\title{
Performance Analysis of Filtered Multitone Modulation Systems for Underwater Communication
}

\author{
João Gomes*† \\ *Instituto Superior Técnico \\ Instituto de Sistemas e Robótica \\ Av. Rovisco Pais, 1049-001 Lisboa, Portugal \\ jpgeisr.ist.utl.pt
}

\author{
Milica Stojanovic ${ }^{\dagger}$ \\ ${ }^{\dagger}$ Northeastern University \\ Department of Electrical and Computer Engineering, 409 DA \\ Boston, MA 02115 \\ millitsalece.neu.edu
}

\begin{abstract}
In Filtered Multitone Modulation (FMT), the bandwidth is split into a number of subbands in which single-carrier signals are modulated onto separate carriers with very little spectral overlap, making the waveform resilient to intercarrier interference. Demodulation is followed by a bank of parallel equalizers, one for each subband. The length of each equalizer is less than what would be needed in a single-carrier system operating over the same total bandwidth. A channel-estimationbased decision-feedback equalization method using a two-step procedure (estimation/fusion) is proposed for FMT. In this structure, channel estimates are used to cancel postcursor intersymbol interference from input signals prior to linear equalization. Parallel channel estimates of adjacent subbands are then fused, i.e. linked to a common underlying multipath model which exploits frequency correlation to improve upon the channel estimates. The sparse nature of underwater acoustic channels may be exploited by setting to zero all but the significant channel taps, thus further relieving equalization of the estimation noise. The performance of underwater FMT is assessed via simulation and using real data transmitted over $800 \mathrm{~m}$ in shallow water at rates of 2-6 kbit/s. The results are compared to OFDM and single-carrier QPSK modulation operating at similar bandwidth efficiencies.
\end{abstract}

\section{INTRODUCTION}

Single-carrier modulation and equalization-based reception has been extensively studied (and used in practice) for highrate underwater communication [1]. However, recent results have demonstrated practical OFDM communication in underwater channels at competitive rates [2], thus generating much interest due to the simplicity of OFDM receivers, the spectral flexibility of this technique, and the potential for efficiently combining it, e.g., with space-time coding using multiple transmitters and receivers [3].

The most appealing feature of OFDM is possibly the simplicity of FFT-based reception using frequency-domain equalization with a single coefficient per subcarrier and the small number of parameters that need to be set a priori. This is to be contrasted with single-carrier modulation and equalization, where the selection of suitable filter lengths and adaptation of a large number of filter coefficients can be quite challenging in practice [1]. These problems become more severe when the communication bandwidth increases, as the multipath results in longer intersymbol interference (ISI) and compensating it requires longer equalizers. But one should keep in mind that OFDM for underwater channels also presents several challenges. Perhaps foremost is the need for a guard interval between OFDM symbols that is lowerbounded by the delay spread of the channel, which can often last several tens of milliseconds. The active symbol duration must be correspondingly increased to attain good transmission efficiency, and intra-symbol channel variations may then induce significant intercarrier interference (ICI).

The above advantages and drawbacks provide motivation for the present work, which examines the feasibility of Filtered Multitone Modulation (FMT) [4], [5] in underwater acoustic communications. FMT is a type of multicarrier modulation that can be seen as a bridge between OFDM and singlecarrier transmission. The basic idea underlying FMT is quite simple: the available bandwidth, $B$, is divided into $M$ disjoint frequency bands of width $B / M$, onto which single-carrier signals are modulated to create a multiband waveform. Raisedcosine-like signaling pulses are used to achieve near-perfect spectral containment in each band, which contrasts sharply with the strongly overlapping sinc pulses (in the frequency domain) of OFDM. Fig. 1 illustrates the difference in pulse shapes used for OFDM and FMT. Each of the FMT subcarrier signals is a single-carrier transmission at $1 / M$ times the rate of a similar one occupying the full bandwidth $B$. The longer signaling interval makes it easier to deal with multipath because ISI is milder, requiring shorter equalizers whose parameters are easier to set. For the same bandwidth, the number of subcarriers in FMT is usually much smaller than in OFDM, which translates into shorter symbol intervals. Wider subcarrier bandwidths and contained spectra with smooth rolloff in FMT make ICI much less severe than in OFDM.

One can thus regard an FMT system simply as a set of parallel single-carrier waveforms that can be processed independently, but a more interesting perspective is to view it as a filterbank/transmultiplexer similar to the one that is used in OFDM. As in OFDM, an FMT transmit/receive filterbank is designed for perfect reconstruction on a distortionless channel, 


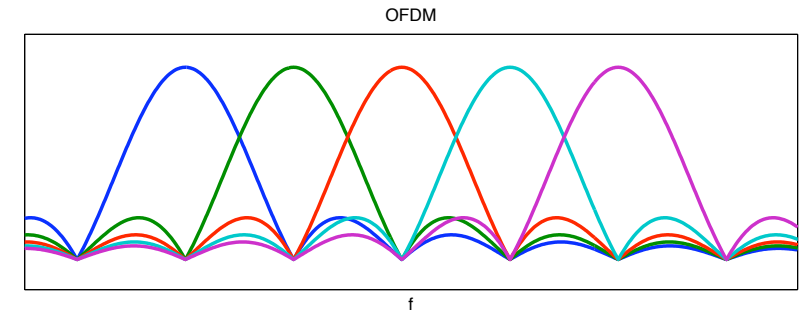

FMT

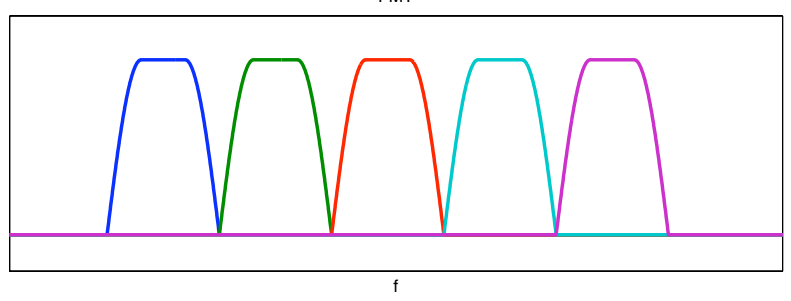

Fig. 1: Subcarrier pulse shapes, in the frequency domain, for OFDM and FMT.

and it can also be implemented using FFT. However, contrary to OFDM, whose spectral characteristics are essentially determined by the signal bandwidth and the number of carriers, in an FMT filterbank there are additional degrees of freedom that enable a tradeoff between spectral containment and the data rate.

In this paper, we focus on post-FFT equalization in FMT systems to compensate for channel-induced ISI. An adaptive structure for decision-feedback equalization (DFE) is proposed, in which the equalizer coefficients are obtained from a channel estimator [6]. This approach allows for channel estimate sparsing, which in turn may improve the equalizer performance. The receiver consists of a separate channel estimator for each subband, and a fusion step that combines them into a single, more reliable estimate. Doppler-induced phase rotations in subcarrier streams are compensated by adaptive phase-locked loops.

The performance of underwater FMT is assessed via simulation, as well as using real data from an experiment conducted in Trondheim, Norway, in September 2007. The data includes several modulation formats with bandwidths of 1.5 and $4.5 \mathrm{kHz}$, recorded at a range of about $800 \mathrm{~m}$ using a 16-hydrophone array. Results are compared to those of conventional OFDM and QPSK with similar spectral efficiency.

The paper is organized as follows. Sec. II discusses FMT modulation and demodulation issues. Sec. III describes equalization algorithms. Sec. IV provides simulation and experimental results that illustrate the performance of FMT. Conclusions are summarized in Sec. V.

\section{FMT MODULATION}

Throughout the paper, vectors and matrices are represented by lowercase boldface and uppercase boldface letters, respectively. The notations $(\cdot)^{T}$ and $(\cdot)^{*}$ stand for transpose and complex conjugate transpose (hermitian), respectively. Convolution is denoted by the binary operator $*$.

An FMT waveform is represented in complex baseband as

$$
x(t)=\sum_{m=0}^{M-1} \sum_{n} a_{m}(n) e^{j 2 \pi \Delta f m t} q(t-n T),
$$

where $M$ is the number of subcarriers, $a_{m}(n)$ is the data symbol transmitted on the $m$-th subcarrier during the $n$-th symbol interval, $q(t)$ is the narrowband pulse shape used in all subcarriers, and $T$ is the signaling interval. For the total available bandwidth $B$, the intercarrier separation equals $\Delta f=B / M$. Fig. 2 shows the baseband FMT filterbank with modulator, channel, and demodulator, represented in discretetime at a sampling rate equal to $B$. As in OFDM, it is possible to efficiently implement the modulator and demodulator of Fig. 2 using the FFT/IFFT, although the processing structures become time-varying [5]. With an ideal channel and properly designed subchannel filters, each output $y_{m}(n)$ should equal a baseband PSK/QAM waveform modulated by the symbol stream $a_{m}(n)$. The following differences between Fig. 2 and an OFDM filterbank should be noted:

- The signaling pulses $q$ are no longer rectangular. They are typically chosen as root-raised-cosine or similar.

- The upsampling/downsampling factor $K$ is greater than the number of subcarriers $M$. This is important for attaining high spectral containment of subcarrier signals.

In principle, one could compute subchannel pulse shapes by quantifying the ICI and ISI at the filterbank outputs and then solving an optimization problem to obtain $q$. This type of approach is pursued e.g. in [7], [8]. In [8], channelindependent ICI metrics are proposed to design FIR filters $q$ by convex optimization, and the intra-channel ISI is then eliminated by post-processing using a single-channel equalizer at each filterbank output (per-subchannel equalization). In the present work we follow the more conventional approach of imposing root-raised-cosine pulse shapes a priori. Our per-subchannel equalization algorithm, discussed in Sec. III, adopts fractional sampling for improved resilience against timing offset errors [9]. Therefore, the filterbank outputs in Fig. 2 are not decimated by $K$, but rather by $K / L$ to attain $L$ samples per symbol interval. ${ }^{1}$

Fig. 3 shows the parameters for one of the raised-cosine subcarrier signaling pulses (including matched filtering by $q^{*}(-t)$ at the receiver). Its total bandwidth equals the subcarrier width, $B / M$. The symbol interval is $T=(1+\beta) /(B / M)$, where $\beta$ is the roll-off factor. In the equivalent discrete-time representation of Fig. 2, one symbol comprises an integer number of $1 / B$-spaced samples, $T=K / B$. This restricts the roll-off factor to values $\beta=K / M-1$. Keeping $0 \leq \beta \leq 1$, i.e. $M \leq K \leq 2 M, K$ can be varied for a given $M$ to obtain

\footnotetext{
${ }^{1}$ Our discrete-time FMT filterbanks actually operate with a sampling frequency that is a multiple of the bandwidth $B$. This avoids having to interpolate the filterbank outputs when $K$ is not a multiple of $L$.
} 


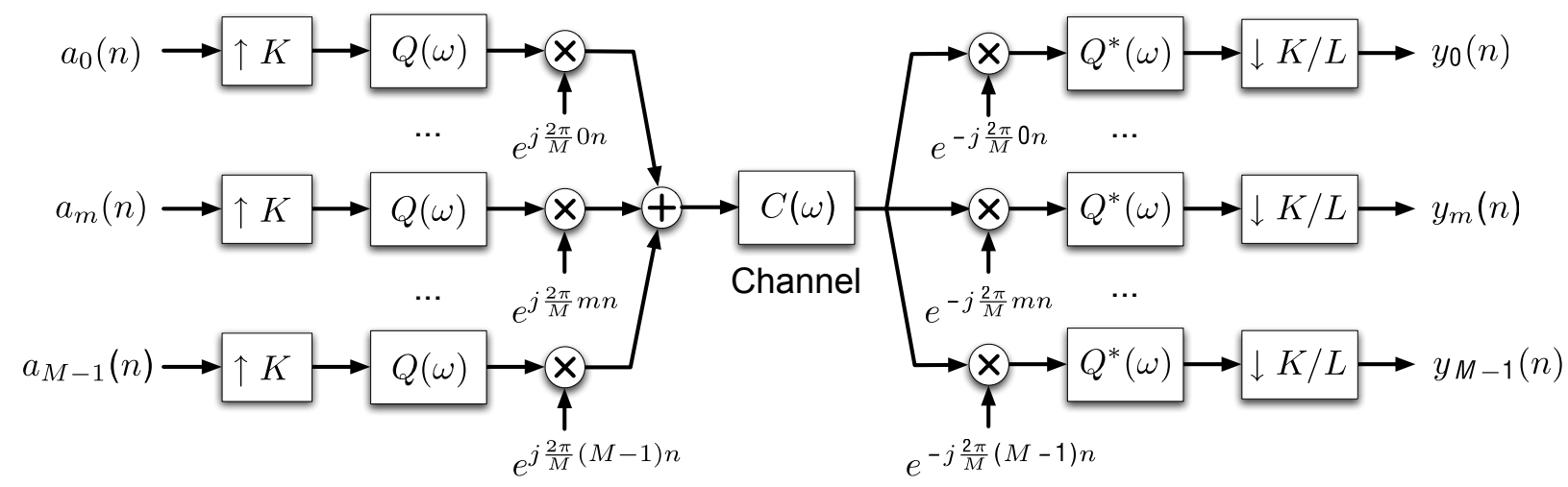

Fig. 2: Discrete-time FMT filterbank (modulator/demodulator).

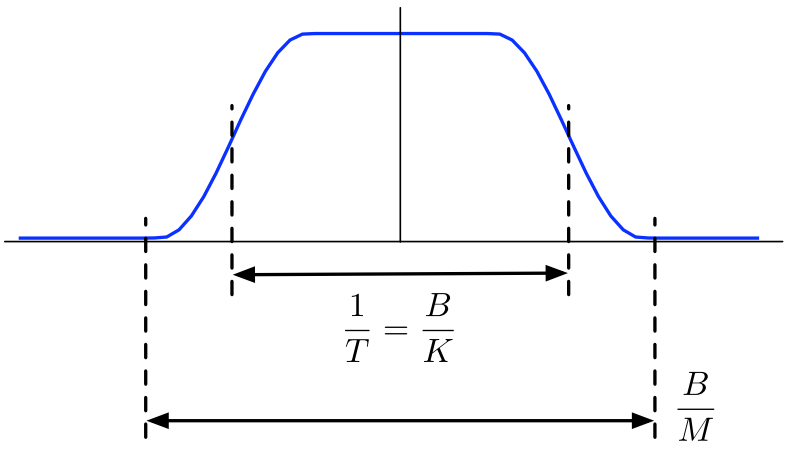

Fig. 3: Parameters of an FMT raised-cosine signaling pulse in the frequency domain.

different roll-off bands and strike a balance between ICI/ISI sensitivity and the data rate.

As there are no guard bands in FMT, either in the time or frequency domains, the spectral efficiency is identical to that of a single-carrier system using a similar signaling pulse with the same roll-off factor over the full bandwidth $\eta=\frac{1}{T B}=\frac{1}{1+\beta}$ sps/Hz. In OFDM, the bandwidth efficiency is $\eta=\frac{1}{1+T_{g} / T}$ sps/Hz, where $T_{g}$ is the length of the guard interval, and $T=$ $1 / \Delta f=M / B$ is the block duration. The relative efficiency of FMT and OFDM depends on the choice of design parameters $\beta$ and $T_{g}$, and the same $\eta$ should be used for fairness when comparing their performances (Sec. IV-A).

By design, FMT symbols overlap partially in time, making it impossible to insert guard intervals between them as in OFDM. This induces an additional difference between these two modulation types:

- The phase terms $e^{j 2 \pi \Delta f m t}$ in (1) should remain continuous between adjacent blocks to preserve the spectral containment of modulated signals. This should be contrasted with OFDM, where similar phase terms are reset in each symbol.

- Phase continuity implies that the filterbank of Fig. 2 is periodically time varying, with a period that depends on the least common multiple of $M$ and $K$. This behavior has to be accounted for when designing a full MIMO equalizer to jointly eliminate ISI in all filterbank outputs. However, it can be ignored in the per-subchannel equalization approach used in this work.

\section{EQUALIZATION IN FMT SYSTEMS}

The addition of zero-prefixes or cyclic-prefixes and guard intervals to isolate OFDM symbols enables FFT-based demodulation with per-subchannel frequency-domain equalization to compensate for the channel distortion. This approach is fundamentally incompatible with FMT, where symbols overlap in time. Instead of scaling each symbol-rate-sampled, ISIfree filterbank output by a single complex coefficient (channel gain), as would be the case in OFDM, a more elaborate filter is needed. The low overlap in frequency that typically exists between adjacent FMT subcarriers for low-to-moderate $M$ even in the presence of realistic Doppler shifts in underwater communications, renders ICI a minor concern when compared with the residual intra-channel ISI. This makes it reasonable to process the filterbank outputs individually at first, using conventional equalizers to eliminate the ISI in each of these signals (they will later be linked to a common multipath structure).

Analysis of single-carrier experimental data has shown that the DFE can provide significant performance gains over linear equalizers, and this structure has become somewhat of a de facto standard in underwater communications [1]. It is adopted in this work as well, for equalization of FMT subchannels. The most straightforward approach is to directly use the multichannel DFE [10], where the feedforward and feedback filters are jointly adapted to minimize the output MSE. This equalization algorithm can accommodate multiple hydrophones and Doppler shifts at the receiver, which remain desirable features for processing FMT signals.

An alternative DFE structure for single-carrier equalization based on direct channel estimation has been proposed in [6]. In this type of DFE, the channel response is tracked explicitly, and it is used directly in a modified feedback filter to subtract the postcursor ISI from input signals based on previous symbol decisions. The relatively mild precursor ISI is then removed by 


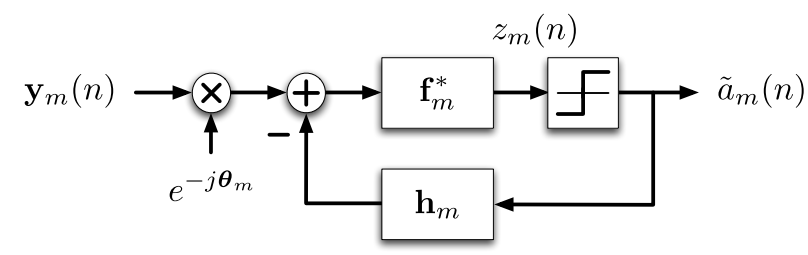

Fig. 4: Channel-estimation-based DFE for one FMT subcarrier stream.

a short linear equalizer. This type of structure readily exploits the sparse nature of many underwater channels, simply by setting to zero those channel coefficients that appear to be negligible. Reduction of the effective number of parameters used by the equalizer can improve the system performance by eliminating some of the estimation noise that is present in all adaptive filtering algorithms.

Fig. 4 broadly outlines the structure of a channel-estimationbased DFE used in each FMT subcarrier stream. In a multichannel configuration, which is often necessary to provide spatial diversity gain in acoustic channels, the receiver filterbank of Fig. 2 is replicated for each hydrophone.

Let $\mathbf{y}_{m r}(n)$ be the vector of input samples to the $m$ th subcarrier equalizer from the $r$-th hydrophone. At time $n$, these vectors include the filterbank outputs $y_{m r}(n-$ $\left.N_{+}\right), \ldots, y_{m r}\left(n-N_{-}\right)$. Phase fluctuations in $y_{m r}(n)$ are compensated by multiplying each sample vector $\mathbf{y}_{m r}(n)$ by an exponential term $e^{-j \theta_{m r}(n)}$. The equalizer is defined by the feedforward filters $\mathbf{f}_{m r}$ and the channel filters $\mathbf{h}_{m r}$. Its output is given by

$$
z_{m}(n)=\sum_{r=1}^{R} \mathbf{f}_{m r}^{*}\left(\mathbf{y}_{m r}(n) e^{-j \theta_{m r}(n)}-\mathbf{y}_{m r}^{b}(n)\right),
$$

where

$$
\mathbf{y}_{m r}^{b}(n)=\sum_{k>0} \mathbf{h}_{m r}(n, k) \tilde{a}_{m}(n-k)
$$

and $\mathbf{h}_{m r}(n, k)$ is obtained from the vector of estimated channel coefficients $\mathbf{h}_{m r}$ in symbol interval $n-k$ by shifting its elements $k L$ times ( $k$ symbols worth of delay). An efficient method for updating $\mathbf{y}_{m r}^{b}(n)$ based on its own shifting property is discussed in [6].

The concatenated feedforward filter coefficient vector, $\mathbf{f}_{m}$, is updated in time by the RLS algorithm [11] driven by the error $e_{m}(n) \triangleq a_{m}(n)-z_{m}(n)$.

The phases $\theta_{m r}$ are adapted so as to approximately minimize the squared error $\left|e_{m}(n)\right|^{2}$, whose gradient is obtained from (2) as [6]

$$
\frac{\partial\left|e_{m}\right|^{2}}{\partial \theta_{m r}}=2 \operatorname{Re}\left\{\frac{\partial e_{m}}{\partial \theta_{m r}} e_{m}^{*}\right\}=-2 \operatorname{Im}\left\{z_{m r}^{f} e_{m}^{*}\right\},
$$

where

$$
z_{m r}^{f}(n)=\mathbf{f}_{m r}^{*} \mathbf{y}_{m r}(n) e^{-j \theta_{m r}(n)} .
$$

The error gradient is used to drive a PLL, normally chosen as a second-order loop [6].
Estimation of the channel vectors used in (3) is carried out in two steps. First, pulse shapes are independently estimated on each subcarrier, and are then fused together so that the $h_{m r}$ in (3) conform to a single underlying channel model (Sec. III-A). Standard system identification techniques are used for the first estimation step [12], in which a bank of parallel identification filters is driven by the sequence of previous symbol decisions (assumed to be correct). Each of these filters is presented with a reference that equals a symbol-rate sampled (and phase corrected) sub-sequence of the receiver filterbank output. Specifically, an RLS update is used for each filter, with input vector

$$
\mathbf{a}_{m}(n)=\left[\begin{array}{lll}
a_{m}(n-N) & \ldots & a_{m}(n-1)
\end{array}\right]^{T}
$$

and reference $y_{m r}\left(\left(n-n_{d}\right) L+l\right) e^{-j \theta_{m r}(n)}, l=0, \ldots, L-1$. The RLS filter for a given $l, m, r$, directly provides a length- $N$ estimate of a symbol-rate-sampled sub-sequence $\hat{h}_{m r}(n L+l)$ for $n=-n_{d}+1, \ldots,-n_{d}+N$. These are interleaved into the correct temporal order for all $l$, yielding length- $N L$ vectors $\hat{\mathbf{h}}_{m r}$. The delay $n_{d}$ in the reference signal must be chosen to capture most of the energy in the causal and anti-causal components of received subcarrier pulse shapes.

Because the same input vector $\mathbf{a}_{m}(n)$ is used in all parallel filters, they also share the computations for updating the data covariance matrix and computing the Kalman gain. In fact, only the recursion for updating the RLS coefficient vector is specific to each filter. Therefore the total cost of performing the proposed $M L R$ identifications is essentially $M$ length- $N$ RLS updates per symbol interval, plus gradient-like updates for the remaining $M(L R-1)$ coefficient vectors.

\section{A. Fusion of Channel Estimates}

The channel estimates $\hat{\mathbf{h}}_{m r}$ are calculated in an efficient manner, but they are obtained independently for each subband $m$. The actual channels, $\mathbf{h}_{m r}$, however, are related to the same physical channel, $C(\omega)$ in Fig. 2. This fact can be exploited to improve upon the channel estimates, and we do so through the process we call fusion.

Consider the path between $a_{m}(n)$ and $y_{m}(n)$ in Fig. 2 . Ignoring the contribution from other branches of the transmit section the signal at the input to the decimator is a discretetime PSK/QAM waveform with $K$ samples per symbol and pulse shape

$$
\begin{aligned}
H_{m}(\omega) & =P(\omega) C\left(\omega+\frac{2 \pi}{M} m\right), \\
P(\omega) & \triangleq|Q(\omega)|^{2},
\end{aligned}
$$

or, in the time domain,

$$
h_{m}(n)=p(n) * c(n) e^{-j \frac{2 \pi}{M} m n} .
$$

The match-filtered pulse above, $p(n) \leftrightarrow P(\omega)$, has a raisedcosine shape. The identification procedure described in the previous section yields $N L$ samples of each received pulse shape $\hat{h}_{m r}$. The delays associated with these samples, relative to the reference sampling rate equal to $B$, will be 
denoted by $n_{1}, \ldots, n_{N L}$. To estimate the channel response for the $r$-th hydrophone in a suitable temporal support region, $c_{r}\left(K_{-}\right), \ldots, c_{r}\left(K_{+}\right)$, all that is needed is to collect the $M N L$ equations

$$
p(n) * c_{r}(n) e^{-j \frac{2 \pi}{M} m n}=\hat{h}_{m r}(n), \quad n=n_{1}, \ldots, n_{N L},
$$

and solve this linear system for $c_{r}$ in an appropriate sense. In standard form:

$$
\begin{gathered}
\mathbf{P c}_{r}=\hat{\mathbf{h}}_{r}, \\
\mathbf{P}=\left[\begin{array}{c}
\mathbf{P}_{0} \\
\vdots \\
\mathbf{P}_{M-1}
\end{array}\right], \quad \mathbf{c}_{r}=\left[\begin{array}{c}
c_{r}\left(K_{-}\right) \\
\vdots \\
c_{r}\left(K_{+}\right)
\end{array}\right], \quad \hat{\mathbf{h}}_{r}=\left[\begin{array}{c}
\hat{\mathbf{h}}_{0 r} \\
\vdots \\
\hat{\mathbf{h}}_{(M-1) r}
\end{array}\right],
\end{gathered}
$$

$\mathbf{P}_{m}=$

$\left[\begin{array}{ccc}p\left(K_{-}-n_{1}\right) e^{-j \frac{2 \pi}{M} m K_{-}} & \ldots & p\left(K_{+}-n_{1}\right) e^{-j \frac{2 \pi}{M} m K_{+}} \\ \vdots & & \vdots \\ p\left(K_{-}-n_{N L}\right) e^{-j \frac{2 \pi}{M} m K_{-}} & \ldots & p\left(K_{+}-n_{N L}\right) e^{-j \frac{2 \pi}{M} m K_{+}}\end{array}\right]$

$$
\hat{\mathbf{h}}_{m r}=\left[\begin{array}{lll}
\hat{h}_{m r}\left(n_{1}\right) & \ldots & \hat{h}_{m r}\left(n_{N L}\right)
\end{array}\right]^{T} .
$$

The most straightforward type of fusion of channel estimates amounts to projecting $\hat{\mathbf{h}}_{r}$ onto the column space of $\mathbf{P}$. If the latter has full column rank this may be accomplished by

$$
\mathbf{h}_{r}=\boldsymbol{\Pi}_{\mathbf{P}} \hat{\mathbf{h}}_{r}, \quad \boldsymbol{\Pi}_{\mathbf{P}}=\mathbf{P}\left(\mathbf{P}^{*} \mathbf{P}\right)^{-1} \mathbf{P}^{*} .
$$

The elements of $\mathbf{h}_{r}$ are then inserted in (3) to synthesize the postcursor ISI waveform. Note that $\mathbf{P}$ does not depend on the hydrophone index $r$, and it may be computed and decomposed as needed offline. When $\mathbf{P}$ is tall, but columnrank-deficient, singular value decomposition (SVD) may be used to compute an orthonormal basis $\mathbf{U}$ for its column space, and the projection operator simply becomes $\Pi_{\mathbf{P}}=\mathbf{U U}^{*}$. Depending on the rank of $\mathbf{U}$, it may be preferable to multiply $\hat{\mathbf{h}}_{r}$ by $\mathbf{U}^{*}$ and $\mathbf{U}$ in sequence, rather than by $\boldsymbol{\Pi}_{\mathbf{P}}$.

For coefficient sparsing as proposed in [6], $\mathbf{c}_{r}$ must first be explicitly computed by solving (11), possibly with some form of regularization. After suitable transformations $\mathbf{c}_{r} \rightarrow \mathbf{c}_{r}^{\prime}$ the fused pulse shapes are obtained as $\mathbf{h}_{r}=\mathbf{P} \mathbf{c}_{r}^{\prime}$, and the vectors $\mathbf{h}_{m r}$ needed for equalization are extracted.

\section{Performance Assessment}

\section{A. Simulation Results}

This section presents simulation results for a deterministic time-varying channel, which were conducted to assess the basic advantages of FMT over the single-carrier and OFDM modulation techniques as discussed in Sec. I. Fig. 5 shows the channel geometry and sound-speed profile, which were chosen to approximate the range-dependent conditions of the UAB'07 experiment described in Sec. IV-B. These simulations focus on the impact of channel variations that cannot be compensated by the phase tracking system described in Sec. III, namely, the presence of differential Doppler that induces distinct frequency shifts in the paths that make up the baseband channel response.

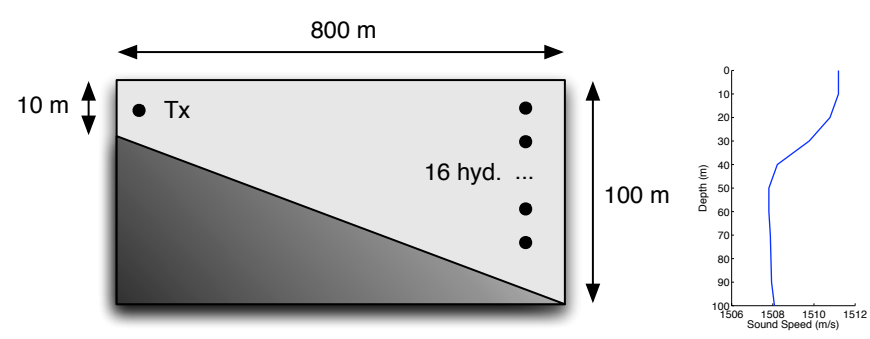

Fig. 5: Simulation setup (geometry and sound-speed profile), reflecting the conditions of the UAB' 07 experiment.

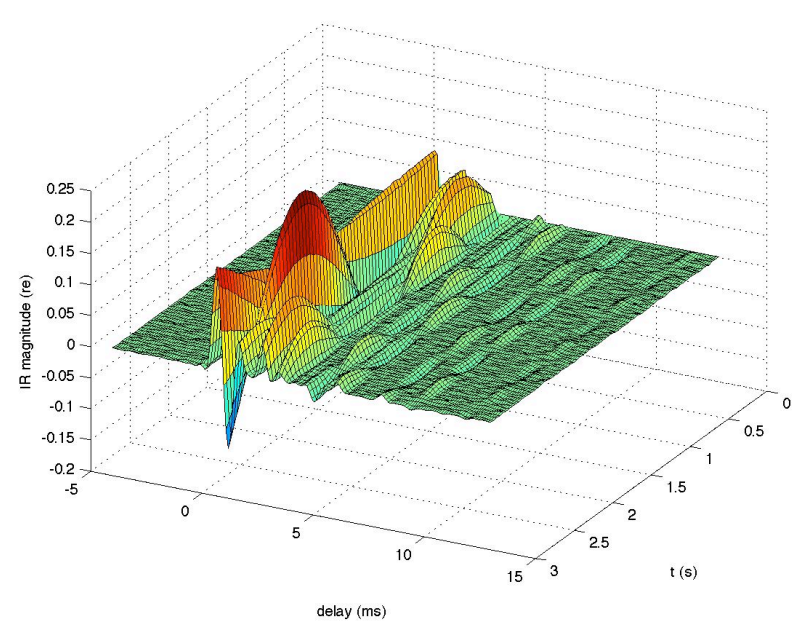

Fig. 6: Time-varying impulse response for simulated channel.

This is accomplished by specifying opposite and vertical-only velocity components for the transmitter and receiver, up to $(0, \pm 0.5) \mathrm{m} / \mathrm{s}$. The communication bandwidth is $B=4.5 \mathrm{kHz}$, around a carrier frequency $f_{c}=5.5 \mathrm{kHz}$.

A soft bottom was used $(v=1530 \mathrm{~m} / \mathrm{s})$, generating an impulse response with delay spread of about $10 \mathrm{~ms}$. Fig. 6 shows the real part of one of the time-varying impulse responses, where the presence of differential Doppler is clear from the difference in phase rotation rates for the various paths. ${ }^{2} \mathrm{~A}$ set of QPSK, FMT and OFDM packets were then generated, distorted by the channel, and demodulated. Their parameters are similar to those used in the UAB' 07 experiment, but they are chosen to ensure that identical spectral efficiencies are used when comparing the performance of different modulations. Specifically, ZP-OFDM packets with 128, 256, 512 QPSKmodulated subcarriers, $25 \mathrm{~ms}$ guard interval and total length of about $3 \mathrm{~s}$ were first specified, yielding bit rates of 4.8, 6.3 and $7.3 \mathrm{kbit} / \mathrm{s}$. For each of these target bit rates, one QPSK packet and 3 FMT packets with 8, 16, 32 carriers were designed by

\footnotetext{
${ }^{2}$ Our approach for incorporating the effects of motion is a coarse approximation, though suitable for testing the performance of different modulations and receivers. Path delays and attenuations were computed for a static nominal configuration using an acoustic ray tracer, and frequency shifts were then computed at the carrier frequency based on departure/arrival angles for each path, and superimposed on the attenuations.
} 

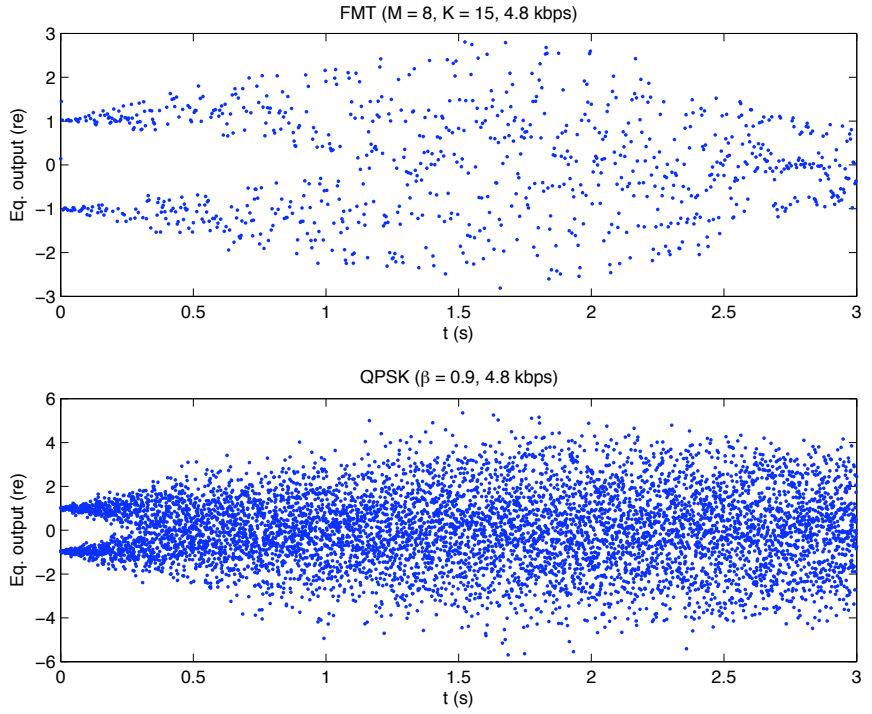

Fig. 7: Real part of equalizer output for one FMT subcarrier $(M=8)$ and a QPSK packet in simulated channel. Clairvoyant receivers are frozen at time $t=0$.

choosing appropriate roll-off factors.

This section focuses on illustrating basic differences in behavior of the above modulations in the presence of channel variations. In order to focus on fundamental differences, i.e. to isolate the artifacts of imperfect tracking by adaptive subsystems (equalization, phase tracking, channel estimation), only clairvoyant receivers are used here. At appropriate instants in time, all required equalizer coefficient vectors or channel gains at OFDM subcarrier frequencies are exactly computed from perfect knowledge of the instantaneous pulse shape/impulse response at any given point in the signal processing chains of the various receivers. Expressions for the coefficients of clairvoyant DFEs are given, e.g., in [13].

Fig. 7 shows the evolution of the real part of the output constellations, after equalization by a DFE, for a $4.8 \mathrm{kbit} / \mathrm{s}$ QPSK packet and the first subcarrier of an FMT packet $(M=8)$. Equalizer parameters were calculated for the instantaneous channel at time $t=0$, and then frozen throughout the packet. The figure clearly shows a significantly faster degradation in the output scatter for the QPSK packet, whose equalizer is longer than in FMT and relies on a delicate balance between its taps to achieve ISI compensation that is more easily disrupted by imperfect channel knowledge. FMT may thus have a practical advantage over QPSK during short-term fast channel fluctuations that exceed the tracking ability of adaptive equalization algorithms at the receiver.

In practice, of course, the receiver parameters will not be frozen during the entire packet but will instead be updated at the symbol rate. The updating rate of a single-carrier system will thus be $M$ times that of an FMT system with the same throughput and efficiency, and so long as the decisiondirected operation remains reliable, channel tracking will be faster. The trade-off between the ISI suppression capability
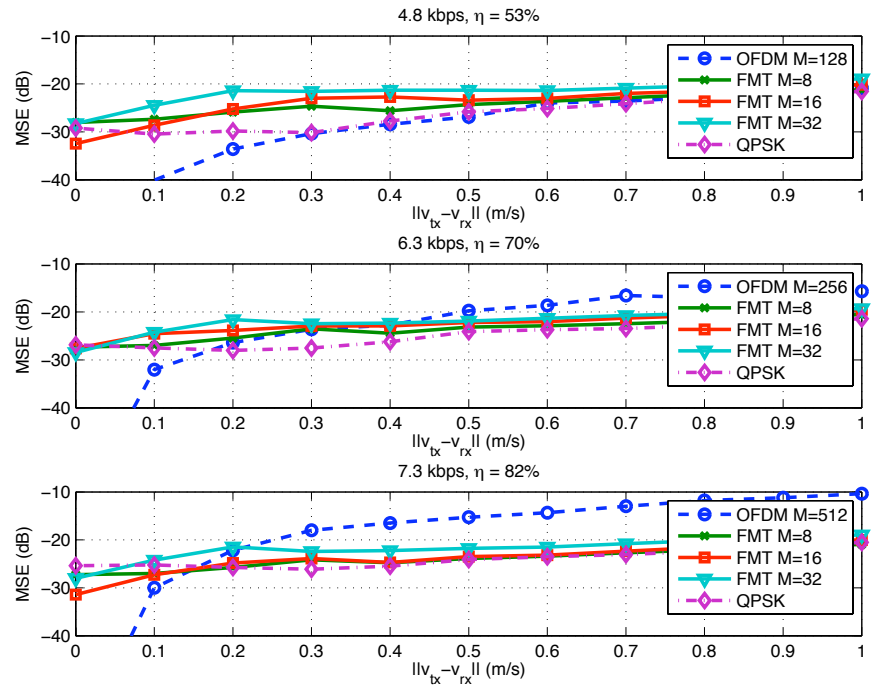

Fig. 8: Output MSE for FMT, QPSK and OFDM packets in simulated channel. Clairvoyant receivers are updated periodically.

and the tracking speed has been addressed in [14], where it was shown that the performance of a single-carrier equalizer improves with increasing bit rate up until a certain point when the bit rate (i.e. updating rate) achieves its full potential. Thereafter, the performance becomes limited by residual ISI. It is precisely at this point that an FMT system could take advantage over a single-carrier system. Namely, one would want to design the system such that the per-subband symbol rate $B / M$ suffices for channel tracking, but then use $M$ adjacent subbands with $M$ parallel equalizers instead of a single band and a single (long) equalizer.

To assess this issue, simulations were conducted in which the clairvoyant receivers were updated periodically throughout a packet. The output MSE for such a strategy is plotted in Fig. 8 vs. the relative transmitter/receiver velocity. OFDM, QPSK and FMT packets were transmitted at the three considered target data rates. No noise was present in the system. Clairvoyant receivers are updated every $50 \mathrm{~ms}$ for the singlecarrier and the FMT systems. For the OFDM systems, the symbol interval can exceed this value, in which case updating occurs at the symbol rate. This is in agreement with basic demodulation methods for OFDM, which do not perform intrasymbol channel tracking. Updating every $50 \mathrm{~ms}$ is sufficiently fast to track channel variations with good accuracy in our simulation scenario, and consequently only small degradations occur in QPSK and FMT as the amount of differential Doppler increases. In OFDM, however, stronger variations occur within one symbol, especially for the larger symbol intervals required at higher data rates, thus causing the MSE to rise. This suggests that FMT may be a better alternative to OFDM under strong channel variations due to finer tracking at the receiver. 


\section{B. Experimental Results}

The experimental data for this section were collected in Trondheim fjord, Norway, during the Underwater Acoustic Barriers (UAB) sea trial in September 2007. The transmit geometry was as depicted in Fig. 5, with the transmitter suspended from a fixed platform $10 \mathrm{~m}$ from shore, at a depth of about $5 \mathrm{~m}$. The receiver was a vertical array with 16 uniformlyspaced hydrophones from $6 \mathrm{~m}$ to $66 \mathrm{~m}$ depth, suspended from a drifting Acoustic Oceanographic Buoy (AOB) developed at the University of Algarve.

QPSK, OFDM and FMT modulations were transmitted with bandwidths of $1.5 \mathrm{kHz}$ and $4.5 \mathrm{kHz}$, and carrier frequency $5.5 \mathrm{kHz}$. Table I summarizes the parameters for QPSK $(\mathrm{Q} n)$, FMT $(\mathrm{F} n)$ and $\mathrm{OFDM}(\mathrm{O} n)$. Each packet is flanked by a pair of start/stop LFM markers used for packet synchronization and coarse Doppler compensation. The transmitter repeatedly cycles through the sequence of packets Q1, F1, F2, F3, O1, O2, O3, Q2, F4, F5, F6, O4, O5, O6. Null subcarriers were inserted in OFDM packets for fine-scale Doppler estimation using the virtual subcarrier (VSC) method [2], and one or two were also included in FMT packets to assess the feasibility of that method. However, due to the high spectral containment and relatively large subcarrier bandwidths it proved to be virtually impossible to detect the small spillover of energy from Doppler shifts into null carriers.

Channel measurements over the full data set at a depth of $22 \mathrm{~m}$ (hydrophone \#5) show two strong and closely spaced arrivals, followed by multiple weaker replicas up to delays of about 20-30 ms. Near the end of the data set one of the late arrivals, at $20 \mathrm{~ms}$, becomes quite strong. Fig. 9a shows the results for channel identification based on a QPSK (Q1) packet (similar to the RLS-based identification method described at the end of Sec. III) in that segment of the data set. Fig. 9b shows a snapshot of the estimated channel for the FMT (F1) packet that immediately follows it, where a similar multipath structure is visible. RLS estimation of subcarrier pulse shapes used $\lambda=0.99$, and regularization through $l_{1}$ norm penalization had to be included in the fusion step of Sec. III-A to overcome ill-conditioning of the linear system (11). This explains why most of the coefficients in Fig. 9b have been automatically set to zero.

Fig. 10 shows performance metrics and sample constellations for demodulation of an F1 packet (\#16). Fig. 10b omits the MSE for $m=6$, which is a null subcarrier. The equalization algorithm uses hydrophones \#2, 6, 10, 14, oversampling $L=2$ per FMT filterbank output, $6 L$ feedforward filter coefficients per hydrophone/subcarrier $\left(N_{-}=-3, N_{+}=2\right)$, and $12 L$ coefficients for pulse shape estimation in the feedback filter $\left(N_{-}=-3, N_{+}=8\right)$. The RLS algorithms for the feedforward and feedback filters use $\lambda=0.995$ and $\lambda=0.99$, respectively. Tracking of each phase $\theta_{m r}$ uses a second-order PLL driven by the phase error (4) with proportional and integral constants $K_{P}=10^{-1}, K_{I}=1.7 \times 10^{-3}$.

Finally, Fig. 11 shows the global MSE for demodulation of four full cycles of consecutive packets Q1-O6, taken from

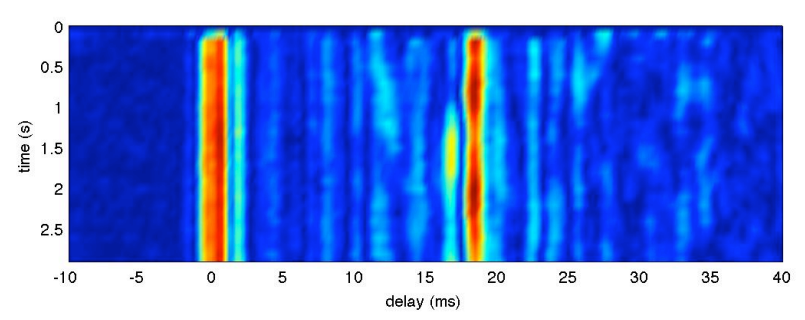

(a)

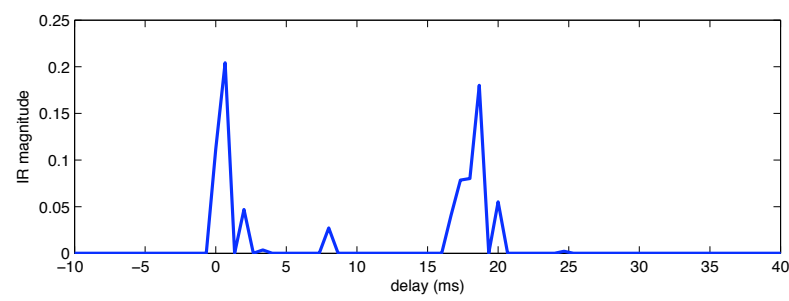

(b)

Fig. 9: Channel identification at depth $22 \mathrm{~m}$ : (a) QPSK packet \#43, (b) FMT packet \#44.

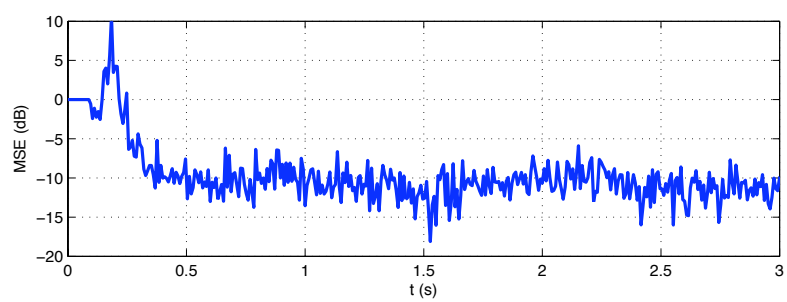

(a)

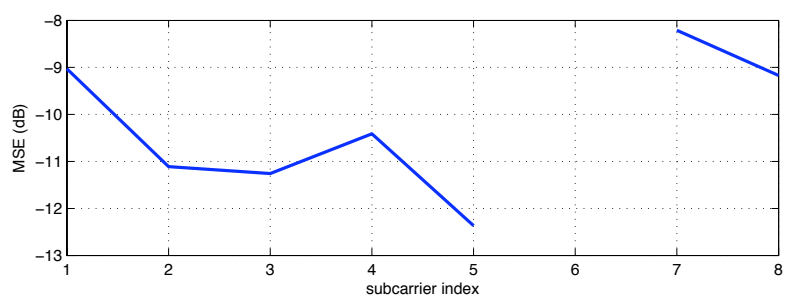

(b)

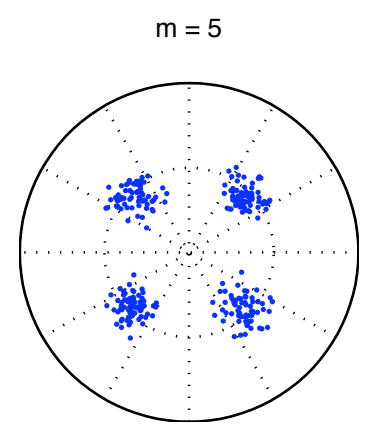

(c)

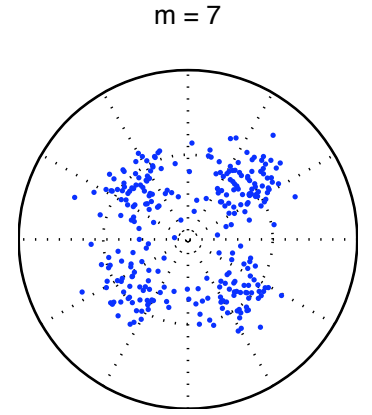

(d)
Fig. 10: Demodulation of FMT packet \#16: (a) Average MSE in time, (b) Average MSE in frequency, (c)-(d) Output constellations for subcarriers 5 and 7. 
TABLE I: QPSK $(\mathrm{Q} n)$, FMT $(\mathrm{F} n)$ and $\mathrm{OFDM}(\mathrm{O} n)$ parameters in the UAB'07 experiment.

\begin{tabular}{|c|c|c|c|c|c|c|c|c|c|c|c|c|c|c|}
\hline Packet type & Q1 & Q2 & F1 & F2 & F3 & $\mathrm{F} 4$ & F5 & F6 & $\mathrm{O} 1$ & $\mathrm{O} 2$ & $\mathrm{O} 3$ & $\mathrm{O} 4$ & $\mathrm{O} 5$ & O6 \\
\hline Bandwidth [kHz] & 1.5 & 4.5 & 1.5 & 1.5 & 1.5 & 4.5 & 4.5 & 4.5 & 1.5 & 1.5 & 1.5 & 4.5 & 4.5 & 4.5 \\
\hline Subcarriers $M$ & 1 & 1 & 8 & 16 & 32 & 8 & 16 & 32 & 64 & 128 & 256 & 128 & 256 & 512 \\
\hline Carrier spacing $[\mathrm{Hz}]$ & \multicolumn{2}{|c|}{ - } & 187.5 & 93.8 & 46.9 & 562.5 & 281.2 & 140.6 & 23.4 & 11.7 & 5.8 & 35.1 & 17.6 & 8.8 \\
\hline Symbol interval $T[\mathrm{~ms}]$ & 1 & 0.3 & 8 & 16 & 32 & 2.7 & 5.3 & 10.7 & 72.7 & 115.3 & 200.7 & 58.4 & 86.9 & 143.8 \\
\hline Guard interval [ms] & \multicolumn{8}{|c|}{$\frac{-}{05}$} & \multicolumn{6}{|c|}{30} \\
\hline Roll-off & \multicolumn{8}{|c|}{0.5} & \multicolumn{6}{|c|}{ - } \\
\hline Null carriers $M_{n}$ & \multirow{2}{*}{\multicolumn{2}{|c|}{ - }} & 1 & 1 & 2 & 1 & 1 & 2 & 3 & 5 & 10 & 5 & 10 & 20 \\
\hline Guard carriers $M_{g}$ & & & \multicolumn{6}{|c|}{0} & 10 & 20 & 40 & 20 & 40 & 78 \\
\hline Number of symbols $N$ & $3 \times 10^{3}$ & $9 \times 10^{3}$ & 400 & 200 & 100 & 1200 & 600 & 300 & 40 & 30 & 15 & 60 & 40 & 20 \\
\hline Constellation & & & & & & & QPSK & & & & & & & \\
\hline
\end{tabular}

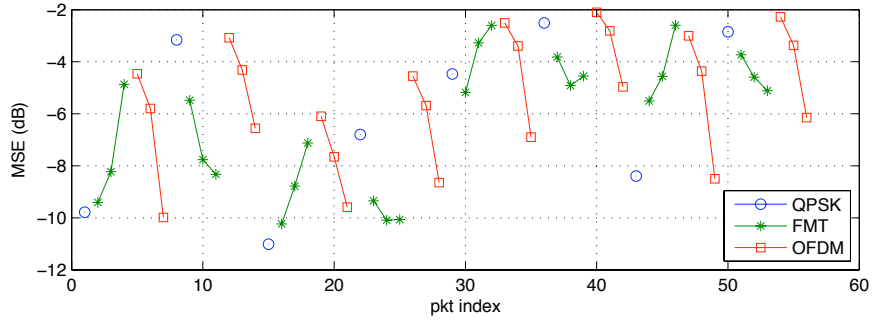

Fig. 11: Demodulation performance over four full packet cycles (Q1-O6).

four different regions of the data set. OFDM demodulation is performed according to [2], using $1 / 4$ of active carriers for channel estimation and 4 hydrophones for multichannel combining. QPSK demodulation uses plain multichannel equalization with 4 hydrophones, $L=2$ oversampling, RLS and PLL parameters as described previously. The lowest MSEs are obtained in Q1 packets using an equalizer with 50 feedback coefficients. To cover the same temporal span Q2 would require a feedback section with some 150 coefficients, but this proved to be unfeasible due to numerical instability. Reducing the feedback filter length (to 70 coefficients) preserves stability, but leads to high MSEs. By contrast, FMT in F1-3 packets performs worse than Q1, but outperforms Q2 in F4-6 because there are no stability vs. accuracy issues in the shorter subcarrier equalizers. Moreover, the figure suggests that it is best to select a low value of $M$ in FMT, say, $M=8$, as a tradeoff for operating on both bandwidths of 1.5 and $4.5 \mathrm{kHz}$. Regarding OFDM, overall its MSE is about $1 \mathrm{~dB}$ higher than FMT. Somewhat surprisingly, best performance is obtained for higher values of $M$, which could be due to better channel estimation (more pilots) under reasonably stable propagation conditions.

\section{CONCLUSION}

Filtered multitone modulation (FMT) was analyzed in the context of underwater acoustic communications. Its performance was assessed through simulated and experimental data analyses, and contrasted to that of single-carrier modulation on the one hand and OFDM on the other. FMT can in fact be regarded as a bridge between these two extreme types of modulation. As such, it stands to gain both the ability to track the time-variation of the channel (which is the main feature of single-carrier wideband modulation) and the simplicity of equalizing only the narrow sub-channels (the advantage of OFDM).

This work focused on a particular channel-estimation-based decision-feedback equalization architecture for FMT, where one can account for the natural sparseness of the channel in a simple way to improve the equalizer performance by selectively truncating some of the estimated channel taps. This equalizer inherits the same benefits of the originally proposed sparsing technique for single-carrier modulation, but has an additional advantage in that it requires a parallel bank of shorter filters, rather than a single long filter. This fact enables a more efficient implementation of the fast-tracking algorithms such as RLS.

Our experimental and numerical results seem to corroborate the notion that by splitting the equalization effort into a bank of short equalizers FMT reduces the numerical issues that hinder the convergence and limit the ISI suppression capability of single-carrier equalizers on underwater channels with long impulse responses. In the data set at hand, this is best accomplished with a small number of subbands, such as 8 or 16 , that reduce the ISI to a manageable number of symbol intervals. Further splitting of the bandwidth may be counterproductive by excessively reducing the ability to resolve multipath components in the time domain.

Simulation results suggest that FMT may outperform OFDM in the presence of channel variations mainly due to finer tracking, but the experimental results are not quite conclusive in this respect, as the channel is apparently stable enough. Overall, FMT does achieve a smaller MSE than OFDM in the UAB'07 experiment, but the difference is only about $1 \mathrm{~dB}$, and the fact that OFDM performance systematically improves as the number of subcarriers increases suggests that the channel is sufficiently stable even over periods in excess of $100 \mathrm{~ms}$. Clarifying this behavior is a topic for future work.

Further work is also needed on sparsing algorithms for the channel-estimation-based DFE. This is most naturally integrated into the fusion step by explicitly computing, and then transforming, a set of channel taps. Low-complexity regularization methods are needed to deal with the numerical ill-conditioning of this problem under certain conditions. 


\section{ACKNOWLEDGMENT}

The work of J. Gomes was supported by Fundação para a Ciência e a Tecnologia through project PTDC/EEATEL/71263/2006, grant SFRH/BSAB/905/2009, and ISR/IST plurianual funding. The work of M. Stojanovic was supported by the ONR MURI Grant \#N00014-07-1-0738.

The authors would like to thank CINTAL and ISR Universidade do Algarve for collecting the experimental data used in this work, the Norwegian University of Science and Technology (NTNU) for the use of the Trondheim marine system research infrastructure (Hydralab III), and R/V Gunnerus master and crew.

\section{REFERENCES}

[1] D. Kilfoyle and A. Baggeroer, "The state of the art in underwater acoustic telemetry," IEEE J. of Oceanic Eng., vol. 25, no. 1, pp. 4-27, Jan. 2000.

[2] B. Li, S. Zhou, M. Stojanovic, L. Freitag, and P. Willett, "Multicarrier communication over underwater acoustic channels with nonuniform doppler shifts," IEEE J. of Oceanic Eng., vol. 33, no. 2, pp. 198-209, April 2008.

[3] M. Stojanovic, "Adaptive channel estimation for underwater acoustic MIMO OFDM systems," in Proc. of the IEEE Digital Signal Processing and Signal Processing Education Workshop (DSP/SPE'09), Marco Island, FL - USA, Jan. 2009.

[4] G. Cherubini, E. Eleftheriou, S. Ölçer, and J. Cioffi, "Filter bank modulation techniques for very high-speed digital subscriber lines," IEEE Communications Mag., vol. 38, no. 5, pp. 98-104, May 2000.
[5] G. Cherubini, E. Eleftheriou, and S. Ölçer, "Filtered multitone modulation for very high-speed digital subscriber lines," IEEE J. on Selected Areas in Communications, vol. 20, no. 5, pp. 1016-1028, June 2002.

[6] M. Stojanovic, "Efficient processing of acoustic signals for high rate information transmission over sparse underwater channels," Elsevier J. on Physical Communication, vol. 1, no. 2, pp. 146-161, June 2008.

[7] M. Wilbur, T. Davidson, and J. Reilly, "Efficient design of oversampled NPR GDFT filterbanks," IEEE Trans. on Signal Processing, vol. 52, no. 7, pp. 1947-1963, July 2004.

[8] B. Borna and T. Davidson, "Efficient design of FMT systems," IEEE Trans. on Communications, vol. 54, no. 5, pp. 794-797, May 2006.

[9] M. Stojanovic, J. Catipovic, and J. Proakis, "Phase-coherent digital communications for underwater acoustic channels," IEEE J. of Oceanic Eng., vol. 19, no. 1, pp. 100-111, Jan. 1994.

[10] - "Adaptive multichannel combining and equalization for underwater acoustic communications," Journal of the Acoustical Society of America, vol. 94, no. 3, pp. 1621-1631, Sept. 1993.

[11] S. Haykin, Adaptive Filter Theory, 4th ed. Englewood Cliffs, NJ: Prentice-Hall, 2002.

[12] J. Gomes, A. Silva, and S. Jesus, "Adaptive spatial combining for passive time-reversed communications," Journal of the Acoustical Society of America, vol. 124, no. 2, pp. 1038-1053, Aug. 2008.

[13] J. Gomes and V. Barroso, "MIMO decision-feedback equalization with direct channel estimation," in Proc. of the 5th IEEE Workshop on Signal Processing Advances in Wireless Communications (SPAWC'04), Lisboa, Portugal, July 2004.

[14] M. Stojanovic, J. Proakis, and J. Catipovic, "Performance of high-rate adaptive equalization on a shallow water acoustic channel," Journal of the Acoustical Society of America, vol. 100, no. 4, pp. 2213-2219, Oct. 1996, part I. 